\title{
RESPUESTA AGRONÓMICA DEL MANDARINO CV. 'CLEMENVILLA' A LA SUPRESIÓN DEL RIEGO DURANTE EL CRECIMIENTO DEL FRUTO
}

\author{
Temnani, Abdelmalek (1). Berríos, Pablo (1). Pérez, David (1). Gil, Ismael (1). Forcén, \\ Manuel (1). Zapata, Susana (1). Pérez-Pastor, Alejandro (2). \\ 1 Doctorando, Departamento de Ingeniería Agronómica, Universidad Politécnica de \\ Cartagena, Cartagena, Región de Murcia, España. abdelmalek.temnani@edu.upct.es \\ 2 Catedrático, Departamento de Ingeniería Agronómica, Universidad Politécnica de \\ Cartagena, Cartagena, Región de Murcia, España. alex.perez-pastor@upct.es
}

\section{Resumen}

La superficie cultivada de mandarino (Citrus reticulata) representa un $37 \%$ de la superficie total de los cítricos en España, que actualmente se cifra en 110.000 ha. La sostenibilidad de la producción de cítricos, en particular, y de la agricultura, en general, se enfrenta a dos desafíos importantes: (i) la fuerte variabilidad en los precios en origen y (ii) el incremento de la escasez de agua. El objetivo principal del trabajo fue evaluar la respuesta agronómica de mandarina 'Clemenvilla' a la supresión del riego durante cada una de las fases de crecimiento del fruto, y determinar su sensibilidad al estrés hídrico, de cara a la aplicación de estrategias de riego deficitario controlado. Se establecieron 4 tratamientos de riego: un control (CTL) regado al $120 \%$ de la ETc durante todo el ciclo del cultivo y 3 tratamientos de supresión del riego, aplicados durante el final de la fase I e inicio de la II (IS-I), la primera mitad de la fase II (IS-II) y la fase III del crecimiento del fruto (IS-III), comprendidas entre 51 a 100, 76 a 105 y 169 a 240 días después de máxima floración (DDMF), respectivamente. Los ciclos de supresión del riego durante las fase inicial y tardía del fruto permitieron reducir el aporte de agua de riego al $50 \%$, alcanzando una integral de estrés hídrico en torno a $45 \mathrm{MPa} \mathrm{día}^{-1}$, sin afectar a la producción ni a la calidad física del fruto; y en el caso de la supresión durante la fase III de crecimiento del fruto permitió incrementar significativamente la productividad del agua de riego.

\footnotetext{
"Abstract"

The area cultivated with mandarin (Citrus reticulata) represents $37 \%$ of the total of Citrus in Spain, which is currently 110,000 ha. The sustainability of citrus production in particular and of agriculture in general is confronted with two major challenges: (i) the strong variability in prices at origin and (ii) the increase of water scarcity. The main objective of the study was to evaluate the agronomic response of 'Clemenvilla' mandarin to irrigation suppression during each of the fruit growth phases, and to determine its sensitivity to water stress, in view of the application of controlled deficit irrigation strategies. Four irrigation treatments were established: a control (CTL) irrigated at $120 \%$ of ETc during the whole crop cycle and 3 treatments of irrigation suppression, applied during the end of phase $I$ and beginning of phase II (IS-I), the first half of phase II (IS-II) and phase III of fruit growth (IS-III), ranging from 51 to 100,76 to 105 and 169 to 240 days after maximum flowering (DAMF), respectively. The irrigation suppression cycles during the initial and late fruit phase allowed reducing the irrigation water use to $50 \%$, reaching an integral of water stress around $45 \mathrm{MPa}$ day $^{-1}$, without affecting the production or the fruit physical quality; and in the case of suppression during the phase III of fruit growth allowed increasing significantly the irrigation water productivity.
} 


\section{1- Introducción}

La superficie cultivada de mandarino (Citrus reticulata) abarca una superficie de 110.000 ha, lo que representa un $37 \%$ de la superficie total de los cítricos en España (Anuario Estadístico de España, 2019). La sostenibilidad de la producción de cítricos, en particular, y de la agricultura, en general, se enfrenta a dos desafíos importantes: (i) la fuerte variabilidad en los precios obtenidos en origen y (ii) el incremento de la escasez de agua, dado que las predicciones climáticas señalan que el déficit de agua para riego y la ocurrencia de eventos climáticos adversos, tales como precipitaciones torrenciales durante el otoño e invierno se incrementarán debido al efecto del cambio climático, lo que podría afectar el crecimiento y desarrollo del fruto por daños directos sobre éste o sobre la infraestructura de riego. Esta realidad hace necesario evaluar el efecto de estrategias de riego que permitan incrementar la resiliencia de los cultivos frente a estos cambios, es decir, aumentar la eficiencia del uso del agua de riego y, a su vez, la competitividad del sector, al reducir, por ejemplo, el coste energético. El riego deficitario controlado (RDC) es una estrategia de riego basada en la reducción del aporte hídrico por debajo de las necesidades hídricas del cultivo, durante aquellos períodos fenológicos, denominados no críticos, en los que esta reducción no afectaría ni a la producción ni a la calidad de la cosecha. Por estos motivos, se hace necesario delimitar tanto la duración como la intensidad del déficit hídrico aplicado a través de la evaluación de la sensibilidad al estrés hídrico de cada una de las fases de crecimiento del fruto.

\section{2- Objetivos}

Evaluar la respuesta agronómica de mandarina 'Clemenvilla' a la supresión del riego durante las fases de crecimiento del fruto, y determinar su sensibilidad al mismo, para incrementar la eficiencia del uso del agua de riego.

\section{3- Materiales y Métodos}

El estudio se llevó a cabo en mandarinas 'Clemenvilla' durante el ciclo 2019-20 en una finca comercial ubicada en la Región de Murcia, España. Las árboles se re-injertaron en 2015 sobre Citrus $\times$ reshni que había sido establecido el año 2000 sobre un marco de plantación de $6 \times 4 \mathrm{~m}$. El programa de fertilización, control de malas hierbas, plagas y enfermedades se realizó según el criterio del agricultor. El sistema de riego consistió de una lateral por hilera de árboles con 4 emisores por árbol, de un caudal de $4.0 \mathrm{~L} \cdot \mathrm{h}^{-1}$.

Se establecieron 4 tratamientos de riego: un control (CTL) regado al $120 \%$ de la ETc durante todo el ciclo del cultivo y 3 tratamientos de supresión del riego, aplicados durante el final de la fase I e inicio de la II (IS-I), la primera mitad de la fase II (IS-II) y la fase III del crecimiento del fruto (IS-III), comprendidas entre 51 a 100, 76 a 105 y 169 a 240 días después de máxima floración (DDMF), respectivamente (Figura 1). En los tratamientos de supresión se restituyó el riego una vez que se alcanzó el valor umbral de - 2,0 MPa de potencial hídrico de tallo a mediodía solar ( $\Psi$ tallo), considerado un estrés hídrico severo en cítricos (Conesa et al., 2014) o una integral de estrés hídrico alrededor de $45 \mathrm{MPa}$ día $^{-1}$. 
AÑO 2019/2020

CONTROL

FASE I

IS-I 49 días

FASE II

IS-II 24 días

FASE III Cosecha

IS-III 71 días

ene. feb. mar. abr. may. jun. jul. ago. sep. oct. nov. dic. ene. feb.

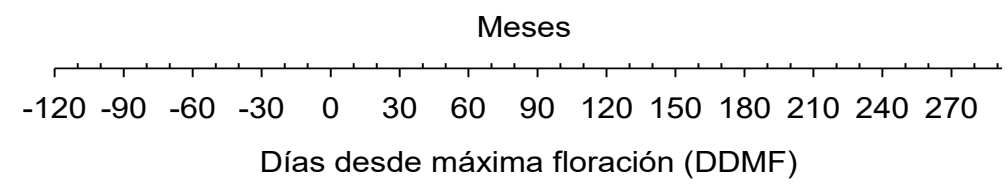

Figura 1. Tratamientos de riego. Control (barra azul) y períodos de supresión total del riego (IS-1: rojo, IS-II: verde e IS-III: amarillo). Las barras horizontales de color gris indican la fase de crecimiento del fruto.

Se realizó un seguimiento a la evolución semanal de $\Psi$ tallo durante mediodía solar con una cámara de presión tipo Scholander modelo Pump-Up (PMS Insrument Company, EE.UU.) en nueve hojas adultas sombreadas por tratamiento. Las hojas fueron cubiertas con una bolsa aluminizada $1.5 \mathrm{~h}$ antes de la medida. La integral de estrés hídrico de cada tratamiento de supresión del riego se determinó a partir de la ecuación 1 (BJ, 1988).

$$
S_{\Psi}=\left|\sum_{i=0}^{i=i}\left(\Psi_{i, i+1}-\Psi_{C T L}\right) n\right|,
$$

Donde: $S_{\Psi}\left(\mathrm{MPa}\right.$ día $\left.{ }^{-1}\right)$, integral de estrés hídrico; $\Psi_{i, i+1}(\mathrm{MPa})$, potencial hídrico de tallo para cada intervalo $\mathrm{i}, \mathrm{i}+1 ; \Psi_{\text {CTL }}(\mathrm{MPa})$, potencial hídrico de tallo correspondiente al tratamiento CTL; y $n$, número de días entre dos medidas consecutivas.

Para el cálculo del área de sección transversal de rama (ASTR) se instaló un dendrómetro perimetral de banda de acero (modelo DB20) por repetición, en la base de una rama principal. EI ASTR se determinó a partir de la ecuación 2.

$$
\operatorname{ASTR}\left(\mathrm{cm}^{2}\right)=\pi\left(\frac{\text { Perímetro }(\mathrm{cm})}{2 \pi}\right)^{2}
$$

La recolección se realizó en todos los árboles evaluados el día 11 de enero de 2020 (262 DDMF) y la producción se determinó como el peso de los frutos comercializables recolectados por árbol y la carga frutal como el número total de frutos. Asimismo, se caracterizó el fruto según su peso medio y diámetro ecuatorial.

La eficiencia en el uso del agua de riego (EUAr, $\mathrm{Kg} \mathrm{m}^{-3}$ ) se determinó como la relación entre la producción por árbol y el volumen de agua de riego utilizado.

Para evaluar la calidad de los frutos, se recolectaron 15 frutos por repetición a los que se les determinó el porcentaje de corteza, pulpa y zumo, y la firmeza exterior. El zumo extraído fue evaluado en base a lo indicado por (Artés et al., 1997), determinándose el pH, sólidos 
solubles (SS), acidez por neutralización con $\mathrm{NaOH} 0.1 \mathrm{~N}$ y el índice de madurez (IM) como la relación entre los sólidos solubles y la acidez.

La evapotranspiración de referencia (ETo) y precipitaciones se obtuvieron a través de la estación MU52 "Cabezo de Plata" perteneciente a la red del Sistema de Información Agrario de Murcia (SIAM, 2019).

Se estableció un diseño experimental con 3 repeticiones por tratamiento distribuidas de forma aleatoria, con 3 árboles por repetición. Se realizó un análisis de varianza y cuando se detectaron diferencias entre tratamientos las medias se separaron mediante la prueba de comparaciones múltiples de Duncan a un $5 \%$ de significancia.

\section{4- Resultados y Discusión}

Durante el período de estudio la ETo acumulada fue de $1377.8 \mathrm{~mm}$ y para los ciclos de supresión de riego correspondió a una media de $6.54,6.56$ y $2.21 \mathrm{~mm}$ día ${ }^{-1}$ para IS-I, II y III; respectivamente. El volumen de riego total aplicado en CTL fue de $5474 \mathrm{~m}^{3}$ por ha y los tratamientos de supresión hídrica permitieron reducir el riego aplicado un 56.7, 55.7 y $52.3 \%$, para IS-I, II y III, respectivamente.

La intensidad del estrés hídrico aplicado expresada como $\mathrm{S}_{\psi}$ fue ligeramente superior en IS-II (49.9 MPa $\cdot$ dia $\left.^{-1}\right)$ y para los tratamientos IS I y III correspondió a 45.2 y $40.7 \mathrm{MPa} \cdot \mathrm{dia}^{-}$ ${ }^{1}$, respectivamente. A pesar de que la demanda climática fue similar durante IS-I e IS-II y la duración del ciclo de supresión de riego durante la fase I fue superior a la fase II, en este último la integral de estrés fue superior puesto que el mínimo $\Psi$ tallo alcanzado en IS-I fue de -1.6 MPa, en contraste a IS-II cuyo mínimo correspondió a -2.1 MPa y fue alcanzado a un menor tiempo después de iniciada la supresión. Asimismo, la tasa de recuperación de $\Psi$ tallo fue superior en IS-I. La menor $S_{\psi}$ alcanzada en IS-III fue dada por la ocurrencia de precipitaciones durante el ciclo de supresión de riego y la menor demanda climática.

Si bien un estrés hídrico severo puede disminuir el crecimiento del tronco o ramas principales (Egea et al., 2009; García-Tejero et al., 2011; E. Pagán et al., 2012) durante el ensayo no se detectaron diferencias entre tratamientos para el incremento del ASTR entre máxima floración y cosecha, aunque CTL mostró un valor promedio superior. La media de incremento correspondió a $0.43 \mathrm{~cm}^{2}$ de ASTR.

Los diferentes parámetros productivos obtenidos no se vieron afectados por efecto de los tratamientos de riego, aunque se observó una cierta variabilidad entre ellos (Tabla 1). Asimismo, tanto el peso como el diámetro del fruto se vieron alterados. Numerosos autores han referenciado la ralentización del crecimiento del fruto ante un estrés hídrico; en este sentido, Pagán et al., (2012) encontraron un descenso significativo en su tamaño final tras someter al mandarino $\mathrm{cv}$. Fortune a un déficit hídrico aplicado durante la fase I y mitad de la II, hasta la ralentización del crecimiento del tronco, que ocasionó una integral de estrés hídrico $\left(\mathrm{S}_{4}\right)$ de $115 \mathrm{MPa}$ día $^{-1}$, mientras que un valor de $55 \mathrm{MPa}$ día $^{-1}$ no implicó reducción alguna. Por ello, en el diseño de estrategias de riego deficitario controlado, no sólo se hace necesario delimitar los períodos no críticos sino también el nivel de estrés hídrico a aplicar. Además, la duración de las restricciones de agua impuestas es determinante en las respuestas de las plantas al RDC (Ginestar \& Castel, 1996).

Así pues, estos resultados se corresponden con otros estudios en los que implementaron una integral de estrés mayor durante la fase II y obtuvieron diferencias no significativas sobre el diámetro ecuatorial de fruto (Dell'Amico Rodríguez et al., 2012). Numerosos estudios muestran la respuesta productiva tras un tratamiento de riego deficitario controlado durante los meses de verano (fase II del crecimiento de fruto), en los que se muestran ahorros de agua en torno a un 15-20\% respecto del control y en los que la producción y la calidad no se vieron afectados (González-Altozano \& Castel, 1999; Elisa Pagán et al., 2012).

Los tratamientos IS-I, II y III permitieron incrementar la eficiencia del uso del agua de riego respecto al control (Tabla 2), con una EUAr muy alta si lo comparamos con otros estudios en los que aplicaron riego deficitario controlado (González-Altozano \& Castel, 1999; Elisa Pagán et al., 2012). 
Respecto a la calidad de los frutos recolectados, el tratamiento IS-II permitió incrementar significativamente la proporción de pulpa y zumo, por lo tanto, disminuir el peso de la epidermis. Este incremento corresponde a un $5.5 \%$ más de rendimiento de pulpa y zumo. No se detectaron diferencias significativas sobre el índice de madurez del fruto entre los ciclos de supresión hídrica respecto a CTL, pero sí entre IS-I e IS-III; esta diferencia viene dada por una menor cantidad de SST obtenidos en IS-III. La supresión en las fases iniciales de crecimiento del fruto (IS-I y II) incrementaron los SST. EI pH del zumo no se vio afectado por ningún tratamiento. Los tratamientos no afectaron significativamente a la firmeza exterior del fruto respecto a CTL, pero sí se detectaron diferencias entre IS I y III (Tabla 2).

Tabla 1. Componentes de rendimiento, productividad y eficiencia del uso del agua de riego.PF: peso del fruto. DEF: diámetro ecuatorial del fruto. CF: carga frutal. ASTR: área sección transversal de rama. EUAr: eficiencia del uso de agua de riego.

\begin{tabular}{|c|c|c|c|c|c|c|c|c|c|c|c|c|}
\hline \multirow{3}{*}{$\begin{array}{c}\text { Tratamiento } \\
\text { CTL }\end{array}$} & \multicolumn{4}{|c|}{ Rendimiento } & \multirow{2}{*}{\multicolumn{2}{|c|}{$\begin{array}{l}\text { Peso } \\
\text { fruto } \\
g\end{array}$}} & \multirow{2}{*}{\multicolumn{2}{|c|}{$\begin{array}{c}\text { Diámetro } \\
\text { ecuatorial } \\
\mathrm{mm}\end{array}$}} & \multirow{2}{*}{\multicolumn{2}{|c|}{$\begin{array}{c}\text { Carga } \\
\text { frutal } \\
\text { Frutos arbol-1 }^{-1}\end{array}$}} & \multirow{2}{*}{\multicolumn{2}{|c|}{$\begin{array}{l}\text { EUAr } \\
\mathrm{Kg} \mathrm{m}^{-3}\end{array}$}} \\
\hline & \multicolumn{2}{|c|}{$\mathrm{Kg}$ árbol-1 } & \multicolumn{2}{|c|}{$\mathrm{t} \mathrm{ha}^{-1}$} & & & & & & & & \\
\hline & 107.5 & a & 48.3 & $a$ & 75.94 & $a$ & 62.79 & $a$ & 1388 & $a$ & 8.19 & $b$ \\
\hline IS I & 97.4 & 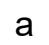 & 45.0 & $a$ & 71.16 & a & 62 & a & 13 & $a$ & 13 & $a b$ \\
\hline IS & 109.3 & $\mathrm{a}$ & 43.3 & $\mathrm{a}$ & 67.10 & a & 59 & a & 1667 & $a$ & 14. & $a b$ \\
\hline $10 \mathrm{III}$ & 123.3 & $\mathrm{a}$ & 45.7 & $\mathrm{a}$ & 71.84 & a & 61.22 & a & 1832 & $\mathrm{a}$ & 17.94 & a \\
\hline
\end{tabular}

Tabla 2. Parámetros de calidad del fruto. SST: sólidos solubles totales. AT: acidez titulable. IM: índice de madurez. Diferentes letras dentro de cada columna indican diferencias significativas según la prueba de Duncan $(p<0.05)$.

\begin{tabular}{|c|c|c|c|c|c|c|c|c|c|c|c|c|c|}
\hline Tratamiento & $\begin{array}{c}\text { Pulpa + } \\
\text { zumo } \\
\%\end{array}$ & \multicolumn{2}{|l|}{$\begin{array}{c}\text { Piel } \\
\% \\
\end{array}$} & \multicolumn{2}{|c|}{ IM } & \multicolumn{2}{|c|}{$\begin{array}{l}\text { SST } \\
{ }^{\circ} \text { Brix }\end{array}$} & \multicolumn{2}{|c|}{$\begin{array}{c}\text { AT } \\
g^{-1}\end{array}$} & \multicolumn{2}{|c|}{$\mathrm{pH}$} & \multicolumn{2}{|c|}{$\begin{array}{c}\text { Firmeza } \\
\text { exterior } \\
\mathrm{Kg} \mathrm{cm}^{-2}\end{array}$} \\
\hline CTL & $59.4 \quad b$ & 40.6 & $a$ & 15.5 & $a b$ & 12.3 & $b$ & 7.9 & $\mathrm{~b}$ & 3.2 & $a$ & 6.2 & $a b$ \\
\hline 15 & 60.1 & 39.9 & a & 16.6 & $a$ & 13.7 & a & 8.3 & $a b$ & 3.3 & a & 6.6 & a \\
\hline IS II & 64.9 & 35.1 & $N$ & 15.3 & $a b$ & 13.9 & $a$ & 9.1 & a & 3.2 & a & 6.3 & $a b$ \\
\hline IS III & 60.2 & 39.8 & a & 14.6 & $b$ & 12.3 & $b$ & 8.4 & $a b$ & 3.2 & a & 5.2 & $b$ \\
\hline
\end{tabular}

\section{Conclusiones}

Los ciclos de estrés hídrico aplicados durante cada una de las fases de crecimiento del fruto de mandarino 'Clemenvilla' supusieron una integral de estrés hídrico inferior a 50 MPa día ${ }^{-1}$. A estos niveles de estrés no se vio afectado ni el rendimiento ni las características físicas del fruto e incluso la supresión de riego permitió incrementar significativamente la eficiencia del uso del agua de riego, aunque todos los tratamientos permitieron ahorrar alrededor de un $50 \%$ del volumen de agua utilizado durante el ciclo de cultivo.

No se vieron afectadas ni la maduración del fruto ni la firmeza exterior respecto al control en ninguno de los tratamientos, pero la supresión de riego durante la fase II de crecimiento del fruto permitió incrementar la proporción de zumo + piel y los sólidos solubles sin afectar el índice de madurez.

Estos resultados deben validarse, al menos, durante un segundo ciclo de crecimiento, comprobando el efecto del estrés hídrico aplicado durante la segunda mitad de la fase II del crecimiento del fruto, incluso estudiar su efecto ante distinta carga frutal, dada la vecería característica de este cultivar.

\section{6-Agradecimientos}

Esta investigación ha sido financiada por dos proyectos, uno en el marco del proyecto europeo DIVERFARMING (H2020-RUR-06-2016) a través del Programa Marco Horizonte 
2020 de la Unión Europea para investigación e innovación y por el Swiss State Secretariat for Education, Research and Innovation, y otro por la convocatoria de ayudas a Acciones Programación Conjunta Internacional 2017 contempladas en el Programa Estatal de I+D+I orientada a los retos de la sociedad por el Ministerio de Economía, Industria y Competitividad - Agencia Estatal de Investigación (AEI). Referencia: PCIN-2017-091.

\section{7-Bibliografía}

Anuario Estadístico de España. (2019). www.ine.es/infoine

Artés, F., Marín, J. G., Martínez, J. A., Tudela, J. A., \& García-Lidón, A. (1997). Desverdización de nuevas variedades de limón y pomelo. Phytoma, 90, 130-135.

BJ, M. (1988). Water Stress Integral-A Link Between Short-Term Stress and Long-Term Growth. Tree Physiology, 4(4). https://doi.org/10.1093/TREEPHYS/4.4.315

Conesa, M. R., García-Salinas, M. D., de la Rosa, J. M., Fernández-Trujillo, J. P., Domingo, R., \& Pérez-Pastor, A. (2014). Effects of deficit irrigation applied during fruit growth period of late mandarin trees on harvest quality, cold storage and subsequent shelf-life. Scientia Horticulturae, 165, 344-351. https://doi.org/10.1016/J.SCIENTA.2013.11.023

Dell'Amico Rodríguez, J. M., Domingo, R., \& Pérez-Pastor, A. (2012). Efecto del estrés hídrico en el desarrollo final del fruto de mandarina 'Fortune.' Cultivos Tropicales, 33, 63-68.

Egea, G., Pagán, E., Baille, A., Domingo, R., Nortes, P. A., \& Pérez-Pastor, A. (2009). Usefulness of establishing trunk diameter based reference lines for irrigation scheduling in almond trees. Irrigation Science, 27(6), 431-441. https://doi.org/10.1007/s00271-0090157-0

García-Tejero, I. F., Durán-Zuazo, V. H., Muriel-Fernández, J. L., \& Jiménez-Bocanegra, J. A. (2011). Linking canopy temperature and trunk diameter fluctuations with other physiological water status tools for water stress management in citrus orchards. Functional Plant Biology, 38(2), 106-117. https://doi.org/10.1071/FP10202

Ginestar, C., \& Castel, J. R. (1996). Responses of young clementine citrus trees to water stress during different phenological periods. Journal of Horticultural Science and Biotechnology, 71(4), 551-559. https://doi.org/10.1080/14620316.1996.11515435

González-Altozano, P., \& Castel, J. R. (1999). Regulated deficit irrigation in "Clementina de Nules" citrus trees. I. Yield and fruit quality effects. Journal of Horticultural Science and Biotechnology, 74(6), 706-713. https://doi.org/10.1080/14620316.1999.11511177

Pagán, E., Pérez-Pastor, A., Domingo, R., Conesa, M. R., \& Caro, M. (2012). Suitability of trunk diameter reference lines for irrigation scheduling with saline water in late mandarin trees with different crop load. Agricultural Water Management, 111, 11-19. https://doi.org/10.1016/j.agwat.2012.04.008

Pagán, Elisa, Pérez-Pastor, A., \& Domingo, R. (2012). Uso de Indicadores del Estado Hídrico de la Planta para la Optimización del Riego en Cultivos Leñosos. Universidad Politécnica de Cartagena.

SIAM. (2019). Informe Agrometeorológico Personalizado. http://siam.imida.es/apex/f?p=101:46:1013612728957675::::: 